\title{
Endometrial and ovarian carcinomas with undifferentiated components: clinically aggressive and frequently underrecognized neoplasms
}

\author{
Laura J Tafe ${ }^{1,3}$, Karuna Garg ${ }^{1,3}$, Ivy Chew ${ }^{1}$, Carmen Tornos ${ }^{2}$ and Robert A Soslow ${ }^{1}$ \\ ${ }^{1}$ Department of Pathology, Memorial Sloan-Kettering Cancer Center, New York, NY, USA and ${ }^{2}$ Department of \\ Pathology, Stony Brook University Medical Center, New York, NY, USA
}

\begin{abstract}
Carcinomas of the endometrium and ovary with undifferentiated components are uncommon neoplasms that are likely underdiagnosed. They are important to recognize as they have been shown to be clinically aggressive. We identified 32 carcinomas with undifferentiated components as defined by Silva and co-workers, 26 endometrial and 6 of ovarian origin. The patient age ranged from 21 to 76 years (median 55); 40\% of patients were $\leq \mathbf{5 0}$ years of age. Most patients $(\mathbf{5 8 \%}$ of endometrial and $\mathbf{8 3} \%$ of ovarian carcinomas with undifferentiated components) presented at advanced stages (FIGO III-IV). Pelvic and para-aortic lymph nodes were the most frequent sites of metastases. Twenty tumors, entirely undifferentiated, consisted of sheets of dyshesive, ovoid cells with uniform, large vesicular nuclei, whereas 12 tumors contained combinations of differentiated endometrioid adenocarcinoma with undifferentiated components. Although most undifferentiated tumors had a monotonous cytologic appearance without prominent stroma, six showed focal nuclear pleomorphism and eight cases had variably sized zones of rhabdoid cells in a background of myxoid stroma. The tumors were frequently misdiagnosed; they received a wide range of diagnoses, including FIGO grade 2 or 3 endometrioid carcinoma, carcinosarcoma, high-grade sarcoma including endometrial stromal sarcoma, neuroendocrine carcinoma, lymphoma, granulosa cell tumor and epithelioid sarcoma. Up to $86 \%$ of the cases showed focal, but strong keratin and/or epithelial membrane antigen staining, with CK18 being the most frequently positive keratin stain. They were predominantly negative for neuroendocrine markers, smooth muscle markers and estrogen receptor/progesterone receptor. Mismatch repair protein expression by immunohistochemistry was evaluated in 17 cases, and 8 (47\%) were abnormal (7 with loss of MLH1/PMS2 and 1 with MSH6 loss). Follow-up was available for 27 patients, although it was very short in many cases, ranging from 0.5 to 89 months (median 9 months). Eleven patients $(41 \%)$ died of the disease in $0.5-20$ months, four are alive with disease and twelve patients have no evidence of disease. Endometrial and ovarian carcinomas with undifferentiated components have a broad histologic differential diagnosis, but they show specific histologic features that should enable accurate diagnosis. These tumors can occur in young women, may be associated with microsatellite instability and behave in a clinically aggressive manner.

Modern Pathology (2010) 23, 781-789; doi:10.1038/modpathol.2010.41; published online 19 March 2010
\end{abstract}

Keywords: endometrium; ovary; undifferentiated carcinoma; endometrioid carcinoma; DNA mismatch repair; malignant mixed Mullerian tumor

Correspondence: Dr RA Soslow, MD, Department of Pathology, Memorial Sloan-Kettering Cancer Center, 1275 York Avenue, MRI1027, New York, NY 10065, USA.

E-mail: soslowr@mskcc.org

${ }^{3}$ These authors contributed equally to this work.

Received 19 August 2009; revised 6 October 2009; accepted 7

October 2009; published online 19 March 2010
Carcinomas of the endometrium and ovary with undifferentiated components are uncommon tumors. The WHO classification describes endometrial undifferentiated carcinomas (UCs) as 'malignant poorly differentiated endometrial carcinomas lacking any evidence of differentiation' without any further characterization. ${ }^{1}$ The lack of a defining set of morphologic features leads to frequent misdiag- 
nosis of these tumors as other more common and specific entities, both in the ovary and endometrium. UCs can also be associated with an endometrioid carcinoma component, and such tumors have been referred to as 'dedifferentiated carcinomas'. ${ }^{2}$ Rather than being only an academic exercise, accurate diagnosis and appropriate classification of these neoplasms are also clinically important, given the prognostic and therapeutic implications. ${ }^{2,3}$ Some of these tumors may also belong to the spectrum of gynecologic neoplasms seen in the setting of microsatellite instability ${ }^{4}$ and, possibly, Lynch syndrome. ${ }^{5}$

All recent studies related to this entity have been from a single large institution. ${ }^{2,3}$ We wanted to review our experience with these rare tumors to study their morphologic and immunohistochemical (IHC) features with particular emphasis on distinguishing them from histologic mimics. Another objective was to explore their relationship with loss of DNA mismatch repair (MMR) proteins. Finally, we sought to assess the clinical characteristics and outcomes of patients with these uterine and ovarian tumors.

\section{Materials and methods}

After obtaining IRB approval, we searched the pathology databases of Memorial Sloan-Kettering Cancer Center and Stony Brook University Medical Center from 2001 to 2008 to retrieve cases of endometrial and ovarian carcinomas with undifferentiated components. We included both pure UCs and UCs associated with a better-differentiated component. The latter tumors have been termed 'dedifferentiated carcinomas' by the MD Anderson group $^{2}$ and will be referred to as 'combined undifferentiated and differentiated carcinomas' in this article. The undifferentiated component of these combined carcinomas is identical to pure UC, and the clinical significance is likely similar. ${ }^{2}$

All H\&E slides were reviewed (2-16 slides per case, median 5). An effort was made to recognize undifferentiated components of combined tumors and pure UCs with a specific histologic appearance; 'UCs' defined solely by the lack of glands or papillae or the presence of anaplasia were not necessarily studied. To distinguish study cases from other uterine tumors with predominantly solid architecture and/or anaplasia, we used the definition proposed by the MD Anderson group ${ }^{2,3}$ for the undifferentiated component, ie, 'a malignant epithelial neoplasm arising in the endometrium or ovary characterized by a total absence of nests, papillae, glands or trabeculae, lack of squamous or mucinous metaplasia, lack of a spindled growth pattern with a patternless solid, sheet-like growth of tumor cells, with absent or minimal neuroendocrine differentiation'. Therefore, the slides were carefully examined to exclude all poorly differentiated tumors that showed specific lines of differentiation, in particular
FIGO grade 3 endometrioid carcinomas and serous carcinomas with a solid growth pattern. In cases where the morphologic appearance evoked the differential diagnosis of neuroendocrine carcinoma, IHC stains for synaptophysin and chromogranin were performed. Tumors that showed diffuse staining (ie strong staining of more than $20 \%$ of tumor cells) for one or both markers were classified as neuroendocrine carcinoma and were excluded from the study.

Both endometrial and ovarian UCs were included in this series as it appears that they are comparable tumors pathologically and clinically, ${ }^{2}$ and perhaps biologically; they can occur in young women and be associated with microsatellite instability. ${ }^{4}$

Immunohistochemical stains, where performed, using standard automated methodology, were assessed (Table 1).

Pertinent clinical information was obtained from the electronic medical records.

\section{Results}

A total of 32 UCs were identified, 26 of the endometrium and 6 of the ovary.

The patient characteristics of our cohort are listed in Table 2. The patient age ranged from 21 to 76 years (median 55 years). Many patients were younger than 50 years of age ( 8 patients $<40$ years $(25 \%)$ and 5 patients were $40-50$ years old (15\%)). Most patients presented with postmenopausal bleeding, vaginal discharge or abdominal pain/discomfort. One young patient presented with seizures and malignant hypertension and was thought to have a paraneoplastic syndrome. Another patient presented with a transient ischemic attack and was found to be coagulopathic with a saddle pulmonary embolus. Two patients had a history of colorectal carcinoma, 4 and 6 years before the endometrial

Table 1 Immunohistochemistry antibodies used in this study

\begin{tabular}{llll}
\hline Antibody & Vendor & Clone & Dilution \\
\hline AE1:AE3 & Ventana & AE1:AE3 & Prediluted \\
CAM 5.2 & Ventana & PD3 & Prediluted \\
EMA & Ventana & MC5 & Prediluted \\
SMA & Ventana & ASM-1 & Prediluted \\
Desmin & Dako & D33 & $1: 50$ \\
ER & Ventana & 6 F11 & Prediluted \\
PR & Ventana & PGR16 & Prediluted \\
S-100 & Dako & RABIT & $1: 500$ \\
HMB-45 & Ventana & HMB-45 & Prediluted \\
CD10 & Ventana & $56 C 6$ & Prediluted \\
Chromogranin & Ventana & LK2H10 & Prediluted \\
Synaptophysin & Ventana & $27 G 12$ & Prediluted \\
CK18 & Dako & DC10 & $1: 200$ \\
Vimentin & Ventana & V9 & Prediluted \\
BAF-47/INI-1 & BD Bioscience & 25 & $1: 100$ \\
MLH1 & BD Bioscience & G168-728 & $1: 1000$ \\
MSH2 & Calbiochem & AB-2 & $1: 200$ \\
MLH6 & BD Bioscience & 44 & $1: 200$ \\
PMS2 & BD Bioscience & A16-4 & $1: 200$ \\
& & & \\
\hline
\end{tabular}


Table 2 Clinical characteristics

Age (years)

Stage at presentation:

Endometrium (26)

Ovary (6)

Synchronous neoplasms in patients

with UC of ovary (number of patients)

History of tumors (number of patients)

Family h/o HNPCC-associated tumors

Clinical outcomes

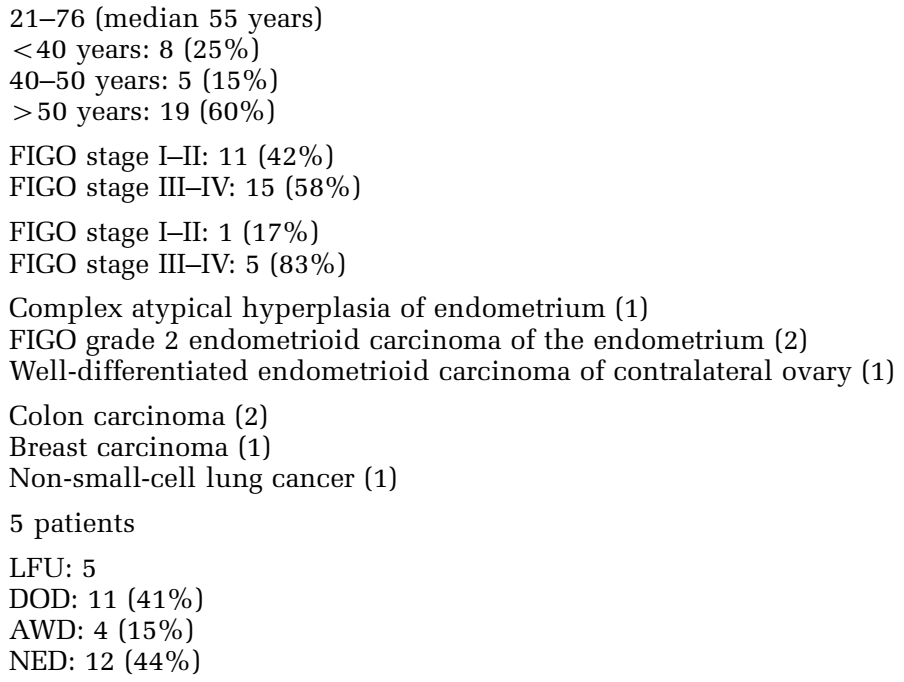

LFU, lost to follow up; DOD, dead of disease; AWD, alive with disease; NED, no evidence of disease.

cancer diagnosis. One patient had a history of invasive ductal carcinoma of the breast and one of non-small-cell lung carcinoma. Of the 32, 5 patients had first-degree family members with a history of Lynch syndrome-associated tumors.

Of the 32 patients, 31 underwent total abdominal hysterectomy and bilateral salpingo-oophorectomy (TAH-BSO). One patient underwent cervical biopsy, and was treated with neoadjuvant chemotherapy, but died of disease and autopsy was performed. Nineteen patients underwent lymphadenectomy. Lymph node metastases were present in 12 of these 19 cases, most frequently involving the para-aortic and pelvic lymph nodes. Of these twelve cases, five also showed pelvic/peritoneal disease, one had liver metastasis and in one case, the tumor also involved the vagina. There were three cases with isolated involvement of other sites (vagina, liver and pelvis/ abdomen), but in two cases lymphadenectomy was not performed.

Of the 26 patients with endometrial UC, 11 (42\%) presented at FIGO stages I-II whereas 15 presented at FIGO stages III-IV (58\%). Lymphovascular invasion was observed in 17 of these 26 cases. Of the six patients with ovarian UC, five $(83 \%)$ presented at advanced stages (stages III-IV) and only one (17\%) presented with stage I disease. One patient had a synchronous well-differentiated endometrioid carcinoma of the contralateral ovary; two patients had synchronous stage I FIGO grade 2 endometrioid carcinomas of the endometrium, whereas one patient had complex atypical hyperplasia of the endometrium.

Most patients with an endometrial primary were treated with adjuvant chemotherapy and/or pelvic or vaginal radiation therapy. All patients with an ovarian primary were treated with chemotherapy alone. Three patients received neoadjuvant chemotherapy before surgery.
Table 3 List of diagnoses rendered for undifferentiated carcinomas of endometrium and ovary

Submitting diagnoses

Number of

cases $(\mathrm{n}=22)$

FIGO grade 2 or 3 endometrioid carcinoma

Poorly differentiated/high-grade carcinoma

Carcinosarcoma

High-grade neoplasm, favor carcinoma

High-grade endometrial stromal sarcoma

Neuroendocrine carcinoma

Adult granulosa cell tumor of ovary

Epithelioid sarcoma of ovary
6

5

4

2

2

1
The follow-up period ranged from 0.5 to 89 months (median 9 months). No follow-up information was available for five patients. Eleven of the remaining 27 patients (41\%) are dead of disease. Four patients are alive with disease $(15 \%$; follow-up 3-7 months) and 12 have no evidence of disease (44\%; follow-up period 6-89 months).

Of the 11 patients who died of disease, 9 had endometrial whereas two had UCs of the ovary. These patients were 21-76 years old (median 56 years), and four of them were younger than 50 years of age. Six of these nine endometrial and both ovarian UCs presented at advanced stages (FIGO stage III-IV). Patients died of disease between 0.5 and 20 months from initial diagnosis (median 6 months).

\section{Tumor Morphology and Immunohistochemistry}

Many of the cases were consult material, which were reviewed at another institution before final diagnosis. The submitting diagnosis was available in 22 of the 32 cases (Table 3). Six cases were 
diagnosed as FIGO grade 2 or 3 endometrioid carcinomas, five as poorly differentiated/high-grade carcinoma, four as carcinosarcoma, one as epithelioid sarcoma, one as granulosa cell tumor of the ovary, two as high-grade endometrial stromal sarcoma, two as high-grade neoplasm favor carcinoma and one as neuroendocrine carcinoma.

Most endometrial UCs presented as large, polypoid masses (size $2-15 \mathrm{~cm}$ ), filling the endometrial cavity. Many tumors showed evident necrosis with diffuse involvement of the endometrium. Although many of the tumors grossly involved the lower uterine segment, two were centered in the lower uterine segment. One of these cases showed two polypoid masses, one centered in the uterine fundus whereas the second originated in the lower uterine segment. Gross cervical involvement by tumor was also frequently noted (10 of 26 cases). The ovarian tumors presented as large tan-brown, fleshy adnexal masses with areas of necrosis and in all the cases, the ovarian surface was involved by tumor.

Of the 32 cases, $20(63 \%)$ were composed purely of UC, whereas the remaining 12 cases (37\%) (10 endometrial and 2 ovarian) showed the presence of an adjacent better-differentiated carcinoma. One endometrial tumor was associated with complex atypical hyperplasia. Two other ovarian UCs were associated with synchronous FIGO grade 2 endometrial endometrioid carcinoma and one with FIGO grade 1 endometrioid carcinoma of the contralateral ovary. In most cases, the better-differentiated component was composed of low-grade (FIGO grade 1 or 2) endometrioid carcinoma, but in two cases, foci of FIGO grade 3 endometrioid carcinoma were also present. In one case, the undifferentiated component was present adjacent to mixed endometrioid and clear cell carcinoma. In all the cases, the transition between the two components was abrupt with a sharp border (Figure 1). Most often, the differentiated component was superficial, whereas the UC was deep to it, although in some cases both components were present adjacent to one another. The amount of differentiated component was variable, but in most cases, it constituted $10-20 \%$ of the total tumor volume, and in one case it was only seen in the biopsy specimen before hysterectomy.

The UC component was characterized by sheets of dyshesive round to ovoid cells, frequently resembling large cell lymphoma, often separated by delicate fibrovascular septa, without any evidence of gland formation, trabecular or nested growth pattern. A few tumors showed foci of vague spindling (Figure 2), although no overtly sarcomatous component was evident. Foci of abrupt keratinization were noted in three cases (Figure 3). Many tumors showed large areas of necrosis with scattered islands of viable perivascular tumor cells (Figure 4).

Tumor cells were round to ovoid with vesicular nuclei and prominent eosinophilic nucleoli. The cells varied from small, basophilic cells with scant cytoplasm to larger cells many of which had clear or

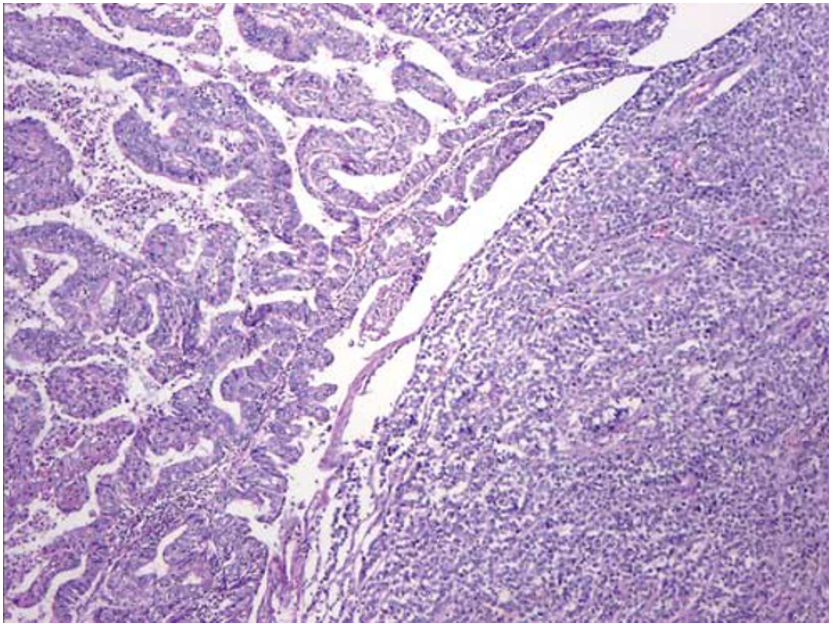

Figure 1 Note the abrupt transition between the differentiated (FIGO grade 1 endometrioid carcinoma) and undifferentiated components in this endometrial dedifferentiated carcinoma.

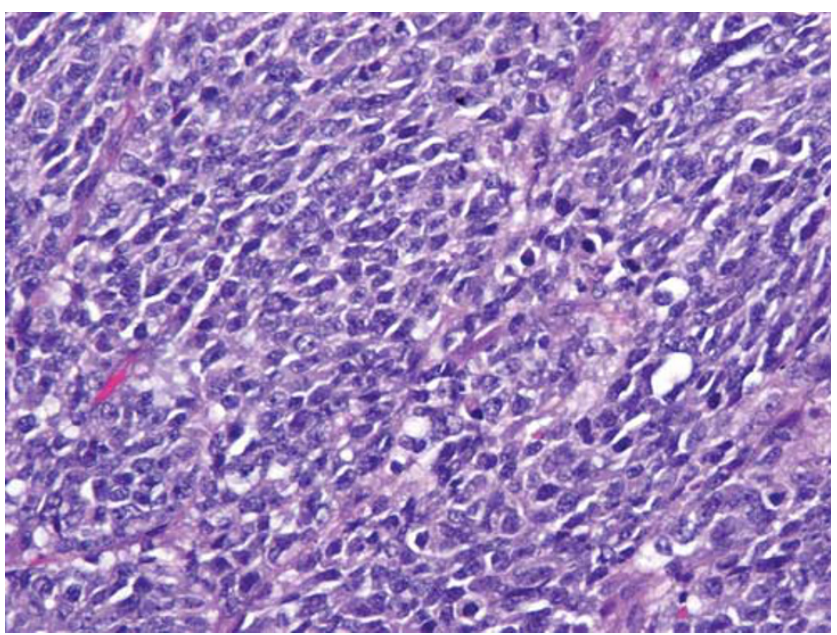

Figure 2 An occasional UC showed areas of vague spindling, but no sarcomatous foci were identified.

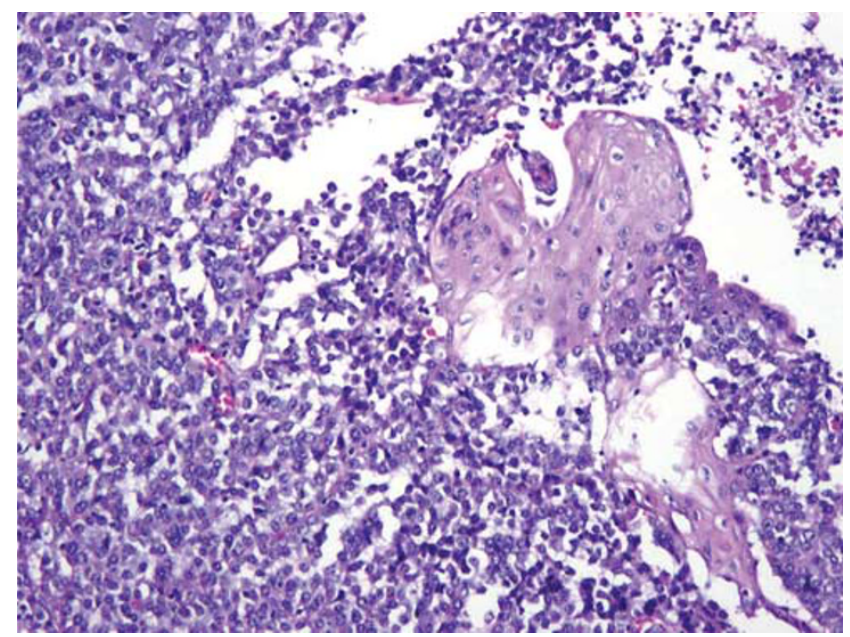

Figure 3 Foci of abrupt keratinization were noted in three cases. 


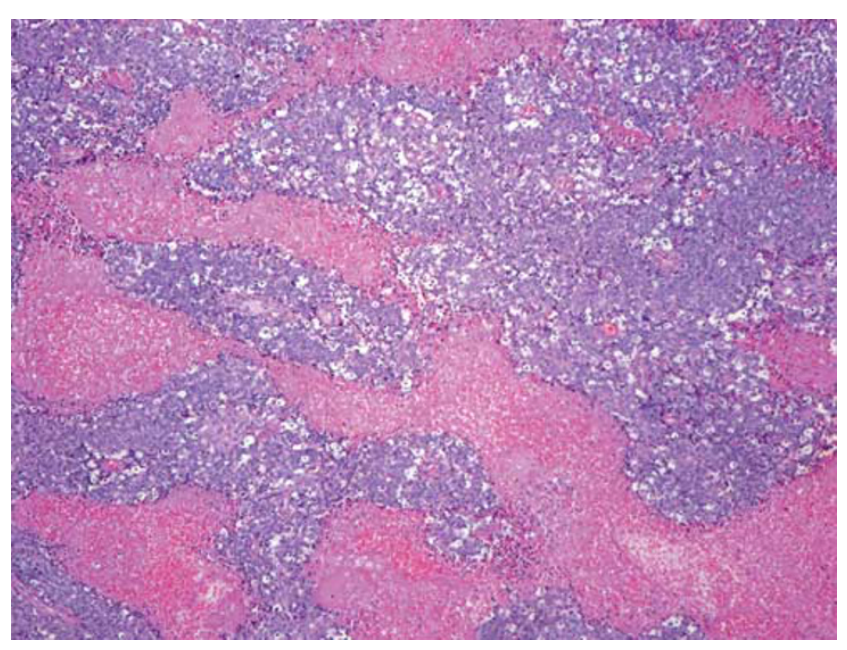

Figure 4 Large areas of necrosis with islands of perivascular viable tumor cells were frequently seen.

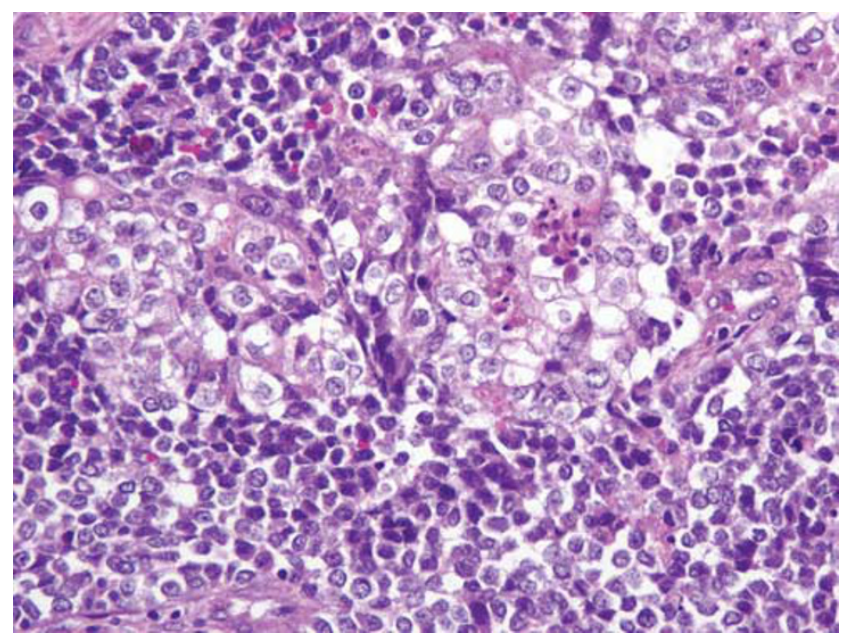

Figure 5 This tumor showed foci with small, basophilic cells with scant cytoplasm and intermingled larger cells with abundant cytoplasm.

vacuolated cytoplasm (Figure 5). Most of the tumors had a monomorphic appearance without marked nuclear pleomorphism $(n=26,81 \%$; Figure 6$)$. A small subset of tumors $(n=6,19 \%)$ showed at least focal areas of marked nuclear pleomorphism in a background of uniform cells as described above. The pleomorphic areas typically contained large cells, many multinucleated, with marked variation in nuclear size and shape, with smudgy and dark chromatin (Figure 7).

Of the 32 tumors, 8 (25\%) showed focal to large areas of rhabdoid cells, ie, large cells with eosinophilic cytoplasm, eccentric nuclei and prominent nucleoli, often in a myxoid background (Figure 8). Many of these rhabdoid areas showed at least focal cord-like growth pattern. Typically, such foci constituted less than $10 \%$ of the total tumor mass.

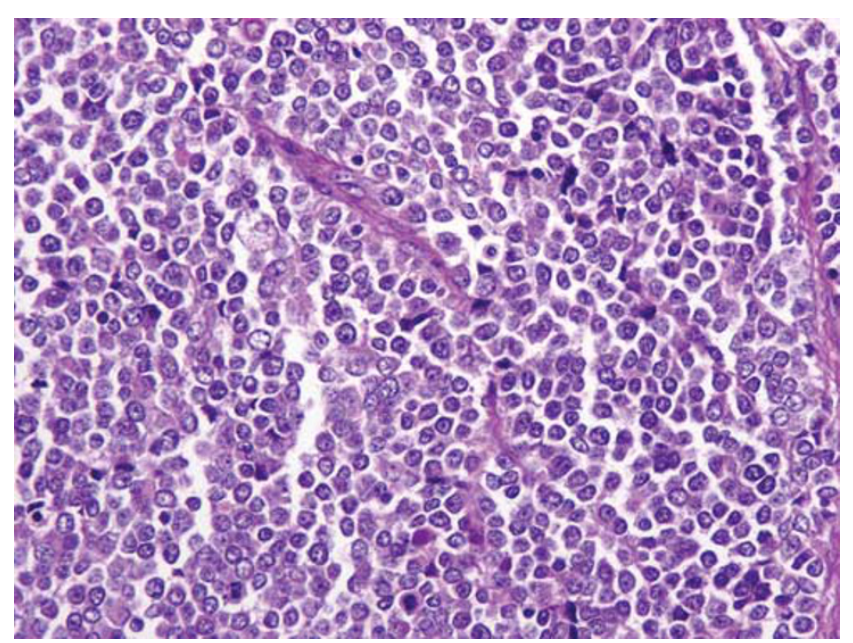

Figure 6 This prototypic undifferentiated carcinoma consists of sheets of dyshesive, ovoid, monotonous cells without evidence of gland formation.

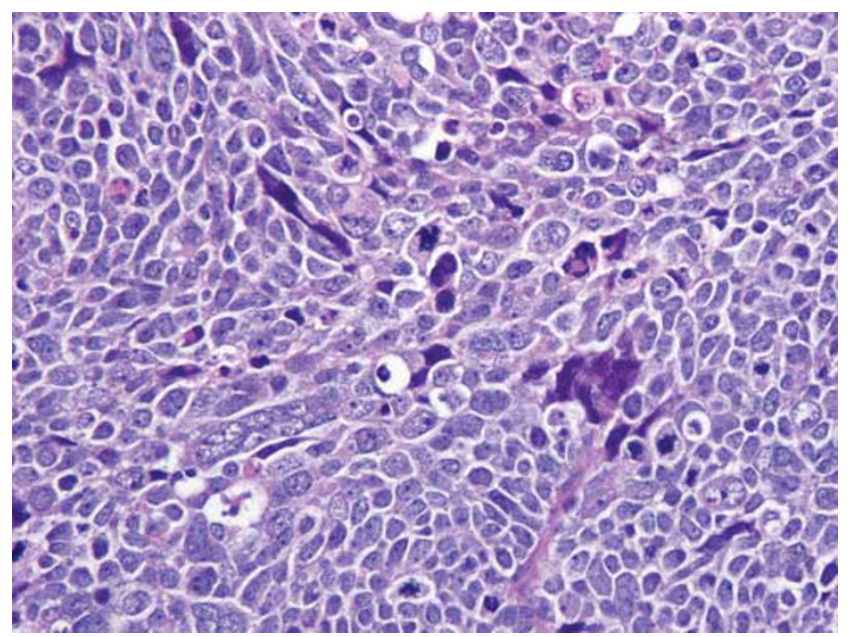

Figure 7 Some of the cases showed marked nuclear pleomorphism, seen here in the form of large multinucleate cells.

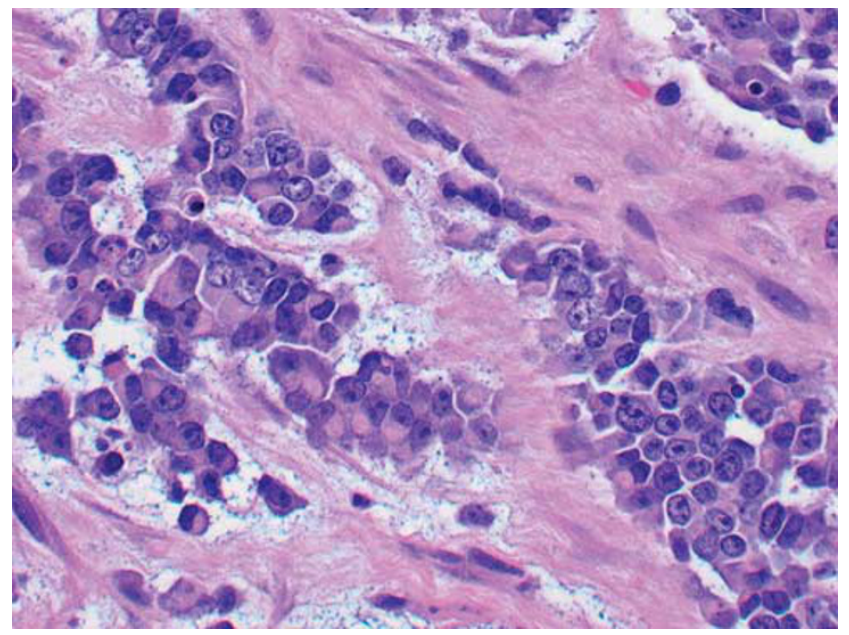

Figure 8 Rhabdoid cells in a myxoid background were noted in few cases. 


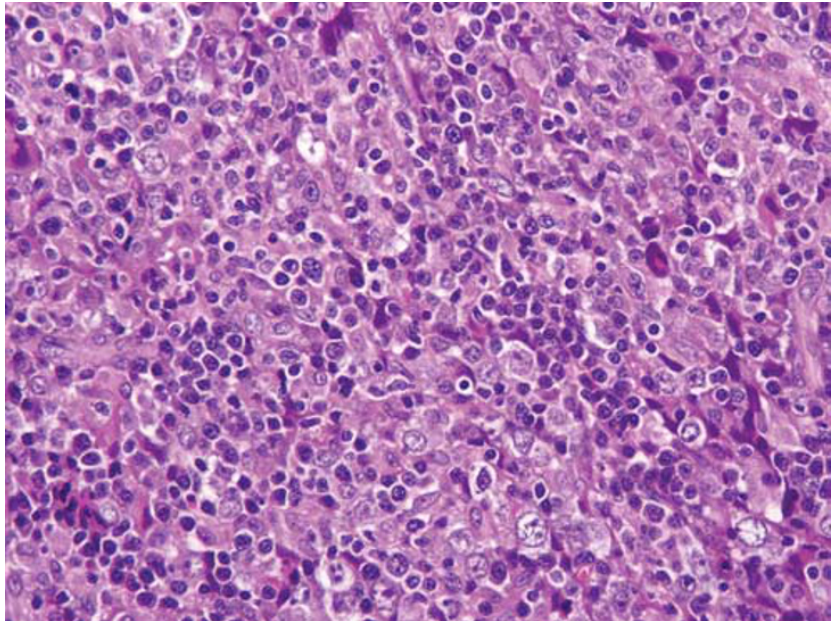

Figure 9 Some UCs showed prominent tumor-infiltrating lymphocytes.

Table 4 Immunohistochemical staining results in undifferentiated tumor components

\begin{tabular}{ll} 
Immunostaining (number of cases) & Results \\
\hline DNA-mismatch repair proteins (17) & MMR loss in 8 cases (47\%) \\
& MLH1/PMS2 loss: 7 \\
& MSH6 loss: 1 \\
Keratin AE1/AE3 (14) & Focal staining in 5 (35\%) \\
CAM 5.2 (15) & Focal in $8(53 \%)$ \\
CK18 (7) & Focal in $6(86 \%)$ \\
EMA (20) & Focal in 16 $(80 \%)$ \\
ER (9) & Focal in $1(11 \%)$ \\
PR (8) & Focal in 1 $(12 \%)$ \\
Chromogranin/synaptophysin (10) & Focal in $3(30 \%)$ \\
BAF-47/INI-1 (3) & Retained nuclear staining \\
& $(100 \%)$
\end{tabular}

The UCs showed frequent mitoses and apoptosis. The mitotic rate varied from 5 to 50 per 10 high power fields (median 18), although most cases showed more than 25 mitotic figures per 10 high power fields, including abnormal mitoses.

Seventeen tumors showed prominent tumor-infiltrating lymphocytes (TILs) ( $>40$ per 10 high power fields), with a median lymphocyte count of 95 per 10 high power fields (Figure 9).

\section{Immunohistochemical Profile}

Immunohistochemical stains were performed in some cases (Table 4). The UC component showed focal (less than $10 \%$ of cells) but intense staining for keratin AE1/AE3 in 5 of 14 cases, keratin CAM 5.2 in 8 of 15 cases and epithelial membrane antigen (EMA) in 16 of 20 cases. CK18 was performed in seven cases, and showed at least focal but intense staining in six cases (Figure 10). The keratin and

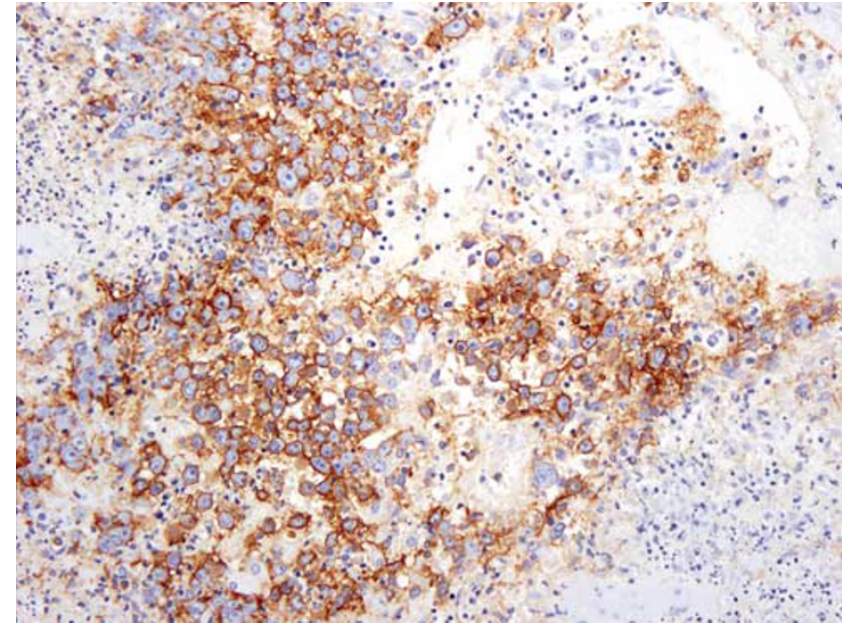

Figure 10 CK18 was the most frequently positive keratin, with focal but strong staining.

EMA stains were more frequently positive in areas with larger cells with more abundant cytoplasm, compared with foci with smaller cells. Estrogen receptor staining (ER) was performed in nine cases and progesterone receptor (PR) in eight cases; all the cases were negative in the undifferentiated component except one that showed focal staining for both ER and PR. The tumors were vimentin positive $(n=3)$. Eight cases were studied for muscle markers, including smooth muscle actin and desmin, and were all negative. BAF-47 (INI-1) was performed in three cases and it showed retained nuclear staining in all three cases, a result expected in tissues with intact INI-1 genes lacking mutation or deletion. Focal S-100 staining was noted in two of seven cases and focal staining for CD10 was also observed in two of seven cases. The tumors were negative for HMB$45(n=6)$. Chromogranin and synaptophysin were performed in 10 cases, with focal $(<10 \%)$ staining in the undifferentiated component for both markers in two cases and focal synaptophysin staining alone in one case. In contrast, the better-differentiated component showed strong staining for all keratins, EMA, ER and PR.

Immunohistochemical staining for DNA MMR proteins (MLH1, PMS2, MSH2 and MSH6) was performed in 17 cases (5 ovarian and 12 endometrial). There was loss of at least one MMR protein in eight cases $(47 \%)$, one endometrial UC showed loss of MSH6 and six showed MLH1/PMS2 loss. One of the five ovarian UCs showed loss of MLH1/PMS2. Six of the seven endometrial UCs with abnormal MMR protein expression were associated with a better-differentiated component, whereas one was not. The ovarian UC with MLH1/PMS2 loss had a synchronous low-grade endometrioid carcinoma of endometrium. The undifferentiated and better-differentiated components showed similar staining patterns for the MMR proteins. 


\section{Discussion}

The MD Anderson group has recently reported that selected UCs have reproducible morphologic features and almost uniformly poor clinical outcomes. ${ }^{3}$ The description of the histologic findings, characterized by sheet-like growth and cytologic uniformity without glandular, papillary or neuroendocrine differentiation, suggests the presence of a specific subset of UCs with distinguishing characteristics. Along these lines, 'UCs' defined solely by the lack of glands or papillae or the presence of anaplasia were not necessarily included for review here. Rather, the presence of a distinctive, patternless proliferation of small- to medium-sized, uniform, dyshesive cells resembling lymphocytes, sometimes with foci of abrupt keratinization, was sought for inclusion in the study. Our report of these endometrial and ovarian carcinomas builds on those from the MD Anderson group ${ }^{2,3}$ and emphasizes these tumors' clinical, morphologic and immunophenotypic distinctiveness. The presence of DNA MMR abnormalities, also previously described by the MD Anderson group, ${ }^{4}$ is also explored in more detail.

Undifferentiated carcinomas can be associated with a differentiated component, most commonly endometrioid carcinoma FIGO grades $1-2 .^{2}$ Twelve of the cases in our series showed these findings and three additional ovarian UCs were associated with synchronous, well- or moderately differentiated endometrioid carcinoma of the uterus or contralateral ovary. We have chosen to label such tumors 'combined undifferentiated and differentiated carcinomas' and recognize that they are part of the spectrum of UC of the endometrium and ovary. The presence of even a small undifferentiated component in tumors with associated differentiated components appears to be associated with poor clinical outcomes. $^{2}$ In our series, we did not note a prognostic difference between such tumors and pure UCs. This underscores the importance of recognizing even a minor component of UC in endometrial and ovarian tumors.

The morphologic appearance of pure UC and combined undifferentiated and differentiated carcinoma evokes a very broad differential diagnosis, which is responsible for frequent misdiagnosis. In the study by the MD Anderson group, UCs were correctly diagnosed in only $18 \%$ of cases. ${ }^{3}$ We also faced frequent difficulties with accurate diagnosis (Table 3).

The main entity usually considered in the differential diagnosis was endometrioid adenocarcinoma, FIGO grade 2 or 3 . A FIGO grade 2 tumor is considered in cases of UC associated with a betterdifferentiated component and FIGO 3 is in the differential diagnosis when the tumor shows a predominantly solid growth pattern. Although FIGO grade 3 endometrioid adenocarcinoma frequently has large areas with a solid growth pattern by definition, it frequently shows glandular or trabecular architecture. The solid components are cohesive and often resemble poorly differentiated, nonkeratinizing squamous cell carcinoma. The nuclear features of tumor cells in both the glandular and the solid areas tend to be cytologically similar. In contrast, the solid areas in UCs are characterized by dyshesive cells that grow in a sheet-like pattern and the cytologic features of the undifferentiated and differentiated components, when present, are distinct. In UCs with a differentiated component, the glands are composed of well-differentiated endometrioid cells whereas the undifferentiated component shows high-grade cytologic features. Rhabdoid foci, which can be seen in a significant proportion of UCs, are not usually seen in endometrioid carcinomas. In difficult cases, IHC stains can be of some assistance as keratins and EMA should show strong diffuse staining throughout FIGO 3 endometrioid carcinomas, whereas in UCs, keratin expression is only patchy and focal in the undifferentiated component. Although FIGO grade 3 endometrioid carcinomas are generally considered high-grade neoplasms, UCs appear to pursue an even more aggressive clinical course. ${ }^{3}$

The well-differentiated component of combined undifferentiated and differentiated carcinoma tends to be superficial, whereas the UC is deep and invasive into myometrium, with an abrupt transition between the two. This very sharp interface prompts consideration of a collision tumor, consisting of a well-differentiated primary and an adjacent poorly differentiated metastatic tumor. None of our patients was found to have another primary tumor.

Serous carcinomas with a solid growth pattern may also enter into the differential diagnosis. Serous carcinomas, even when predominantly solid, usually show at diagnostic of classical serous carcinoma. The presence of papillae and micropapillae, slit-like compressed spaces, background endometrial atrophy and architectural-cytologic dyssynchrony can also provide clues to the correct diagnosis. UCs lack these features.

When associated with a well-differentiated component, UCs are often misdiagnosed as carcinosarcoma (malignant mixed Mullerian tumor) given their biphasic appearance. However, the glandforming components of the former are endometrioid and low grade. Carcinosarcomas, however, usually contain high-grade carcinoma, most frequently serous carcinoma. ${ }^{6}$ Also, UC consists of ovoid, epithelioid cells unlike the sarcomatous component of a carcinosarcoma, which typically consists of a pleomorphic spindle-cell proliferation. Carcinosarcomas typically occur in older women whereas UCs can occur in very young patients. The biology of the two tumors may also be distinct. At least a proportion of UCs appear to be related to microsatellite instability. ${ }^{5}$ Although carcinosarcomas can be seen in the setting of microsatellite instability, it is rare. ${ }^{5}$ 
Two tumors in our series were diagnosed as highgrade endometrial stromal sarcomas, perhaps due to a uniform but high-grade appearance of tumor cells. Tumors previously diagnosed as high-grade endometrial stromal sarcoma are now classified as undifferentiated endometrial sarcomas. In a recent study about tumors classified as undifferentiated endometrial sarcomas at MSKCC, we found one case of UC. ${ }^{7}$ Undifferentiated endometrial sarcomas are frequently much more pleomorphic and at least focally spindled, although a variant with uniform cells has recently been described. ${ }^{8}$ Another helpful hint can be the pattern of metastasis. UCs frequently show lymph node involvement, as seen in our series, in contrast with undifferentiated endometrial sarcomas where lymph node involvement, particularly without extrauterine disease, is very unusual.

The tumor morphology, particularly given the dyshesive growth pattern, can be reminiscent of lymphoma or in cases with a prominent rhabdoid component, plasmacytoma may be a consideration. From a clinical perspective as well, the finding of explosive lymphadenopathy and elevations in serum LDH levels make lymphoma a diagnostic consideration. The possibility of a lymphoid neoplasm can be easily excluded by using the appropriate IHC stains. One should remember that CD138 is frequently positive in various nonhematopoietic tumors including carcinomas of various types, ${ }^{9}$ including those with plasmacytoid morphology. ${ }^{10}$

UCs that show prominent rhabdoid cells can also raise the consideration of an extrarenal malignant rhabdoid tumor, characterized by biallelic deletion or mutation involving the SMARCB1/INI-1 gene on chromosome 22, resulting in loss of IHC expression of INI-1. ${ }^{11}$ A related theoretical consideration would be that of epithelioid sarcoma; one ovarian UC in our series was diagnosed as epithelioid sarcoma at another institution. The BAF-47 (INI-1) stain showed retained nuclear staining in our cases where performed. This result is expected in tissues with intact INI-1 genes lacking mutation or deletion. Retained INI-1 staining has also been reported in a variety of uterine tumors composed of rhabdoid cells, variously classified as carcinosarcoma, sarcoma and uterine tumor resembling ovarian sex cord tumor. ${ }^{12,13}$ We suspect that some of these and other cases reported in the literature as uterine rhabdoid tumors may represent uterine UCs. ${ }^{13-15}$ However, one endometrial tumor reported as a 'composite rhabdoid tumor of the endometrium' (resembling UC with a better-differentiated component in the published illustrations) showed loss of IN1-1 staining. ${ }^{16}$

Another diagnostic consideration is that of combined adenocarcinoma and neuroendocrine carcinoma or pure neuroendocrine carcinoma. We do not diagnose cases with obvious architectural and nuclear features of neuroendocrine differentiation as UCs. UCs can show focal neuroendocrine features and staining with neuroendocrine-related markers without any impact on clinical outcome. ${ }^{17}$ In our study, 3 of 10 cases showed focal (less than 10\%) synaptophysin and/or chromogranin staining. We would classify a tumor that shows strong and diffuse staining for neuroendocrine markers as a neuroendocrine carcinoma rather than UC.

In the ovary, particularly in tumors composed of smaller cells with scant cytoplasm, the morphologic appearance can raise the differential diagnosis of other small round blue cell tumors. One case was submitted to us with a possible diagnosis of granulosa cell tumor. Differentiation from adult granulosa cell tumor can be achieved by examining the nuclear details; whereas UCs are composed of round to ovoid nuclei with vesicular chromatin and frequent nucleoli, the nuclei of adult granulosa cell tumor are frequently long and grooved. Juvenile granulosa cell tumor may be considered, but the lack of follicle-like spaces is helpful. Other considerations may include small-cell hypercalcemic carcinoma, peripheral and central-type primitive neuroectodermal tumor and desmoplastic small round cell tumor. Use of appropriate immunohistochemistry stains and/or molecular studies can resolve this differential diagnosis.

The difficulty in diagnosis is further compounded by the near-complete absence of staining for epithelial markers in UCs. The better-differentiated component, when present, is typically strongly positive with keratins, EMA, ER and PR. In contrast, the adjacent UC can show almost complete loss of expression of these markers. UCs often show only focal staining for keratins and EMA. ${ }^{3}$ In our experience, CK18 is the most helpful stain to show epithelial differentiation in this setting. Sometimes, staining additional blocks for keratins, particularly CK18, and EMA, can help support epithelial differentiation in reaching the correct diagnosis. The possibility of UC must be kept in mind in the setting of a hard-to-classify endometrial or ovarian neoplasm composed of sheets of epithelioid cells that are negative for epithelial markers.

These tumors can show loss of MMR proteins, most frequently MLH1 and PMS2, but sometimes MSH2/MSH6. Although many tumors appear to be associated with MLH1 promoter hypermethylation, ${ }^{4}$ we believe that at least a proportion of UCs are associated with hereditary nonpolyposis colorectal cancer or Lynch syndrome. ${ }^{5}$ Two of our patients had a history of colon cancer, one with MLH1/PMS2 loss. Whereas immunohistochemistry for DNA MMR proteins was not performed in the second patient, the patient met Amsterdam criteria for Lynch syndrome. One patient's tumor showed loss of MSH6 staining, suggestive of Lynch syndrome. In combined undifferentiated and differentiated carcinomas, immunohistochemistry for DNA MMR proteins showed similar staining patterns in both the components, supporting that they have the same origin. One could speculate that a tumor with microsatellite instability, being more prone to 
replication errors, can develop additional genetic abnormalities with progression to an undifferentiated component. Although UCs may be sporadic and associated with MLH1 promoter hypermethylation, it is emphasized that testing all UCs for defective DNA MMR contributes to the recognition of patients at highest risk for Lynch syndrome.

Similar to the MD Anderson group's findings, UCs in our series pursued an aggressive clinical course. ${ }^{3}$ In our cohort, $41 \%$ of patients died of disease, including many young patients, with a fulminant clinical course and very short survivals (range of 0.5-20 months, median 6 months). The apparent differences in the clinical outcomes, with $44 \%$ of our patients having no evidence of disease at last follow-up, is likely due to the fact that a significant number were followed for only relatively short periods of time (mean follow-up in our study was 20 months compared with 70 months in the Altrabulsi et al study). ${ }^{3}$ Our study population also appears to have more young patients and therapeutic approaches may have differed. We could not find any association between stage, age, presence and number of TILs, histologic features (including dedifferentiated histology, presence of pleomorphism or rhabdoid foci) and clinical outcomes. In particular, presence of a better-differentiated component, irrespective of its amount, does not appear to confer improved clinical outcomes.

In summary, we present the clinicopathologic features of 32 cases of UCs of the endometrium and ovary, clinically aggressive and frequently misdiagnosed tumors. We confirmed initial observations by Silva and co-workers ${ }^{2,3}$ and expanded on them. The study emphasizes distinctive clinical, morphologic and immunophenotypic features of these tumors and explores the association with DNA MMR abnormalities. Consideration should be given to UCs a specific entity in the classification of ovarian and endometrial carcinomas.

\section{Disclosure/conflict of interest}

The authors declare no conflict of interest.

\section{References}

1 Tavassoli FA, Devilee P. World Health Organization Classification of Tumors: Tumors of the Breast and Female Genital Organs. IARC Press: Lyon, 2003.

2 Silva EG, Deavers MT, Bodurka DC, et al. Association of low grade endometrioid carcinoma of the uterus and ovary with undifferentiated carcinoma: a new type of dedifferentiated carcinoma? Int J Gynecol Pathol 2006;25:52-58.

3 Altrabulsi B, Malpica A, Deavers MT, et al. Undifferentiated carcinoma of the endometrium. Am J Surg Pathol 2005;29:1316-1321.

4 Broaddus RR, Lynch HT, Chen LM, et al. Pathologic features of endometrial carcinoma associated with HNPCC. Cancer 2006;106:87-94.

5 Garg K, Leitao M, Kauff N, et al. Selection of endometrial carcinomas for DNA mismatch repair protein immunohistochemistry using patient age and tumor morphology enhances detection of mismatch repair abnormalities. Am J Surg Pathol 2009;33:925-933.

6 Ferguson SE, Tornos C, Hummer A, et al. Prognostic features of surgical stage I uterine carcinosarcomas. Am J Surg Pathol 2007;31:1653-1661.

7 Garg K, Leitao M, Guile M, et al. Undifferentiated uterine sarcomas: a diagnosis of exclusion with apparent prognostic significance. Mod Pathol 2008;21:225A.

8 Kurihara S, Oda Y, Ohishi Y, et al. Endometrial stromal sarcomas and related high grade sarcomas: immunohistochemical and molecular genetic study of 31 cases. Am J Surg Pathol 2008;32:1228-1238.

9 O'Connell FP, Pinkus JL, Pinkus GS. CD138 (Syndecan-1), a plasma cell marker: immunohistochemical profile in hematopoietic and nonhematopoietic neoplasms. Am J Clin Pathol 2004;121:254-263.

10 Nigwekar P, Tamboli P, Amin MB, et al. Plasmacytoid urothelial carcinoma: detailed analysis of morphology with clinicopathologic correlation in 17 cases. Am J Surg Pathol 2009;33:417-424.

11 Hoot A, Russo P, Judkins AR, et al. Immunohistochemical analysis of h-SNF5/INI-1 distinguishes renal and extra-renal malignant rhabdoid tumor from other pediatric soft tissue sarcomas. Am J Surg Pathol 2004;28:1485-1491.

12 Al-Hussaini M, Hirschowitz L, McCluggage G. Uterine neoplasms composed of rhabdoid cells do not exhibit loss of INI1 immunoreactivity and are not related to childhood malignant rhabdoid tumor. Int J Gynecol Pathol 2008;27:236-242.

13 Cho KR, Rosenshein NB, Epstein JI. Malignant rhabdoid tumor of the uterus. Int J Gynecol Pathol 1989;8:381-387.

14 Gaertner EM, Farley JH, Taylor RR, et al. Collision of uterine rhabdoid tumor and endometrioid adenocarcinoma: a case report and review of the literature. Int J Gynecol Pathol 1999;18:396-401.

15 Mount SL, Lee KR, Taatjes DJ. Carcinosarcoma (malignant mixed Mullerian tumor) of the uterus with a rhabdoid tumor component. An immunohistochemical, ultrastructural and immunoelectronmicroscopic case study. Am J Clin Pathol 1995;103: 235-239.

16 Donner LR, Wainwright LM, Zhang F, et al. Mutation of the INI1 gene in composite rhabdoid tumor of the endometrium. Hum Pathol 2007;38:935-939.

17 Taraif SH, Deavers MT, Malpica A, et al. The significance of neuroendocrine expression in undifferentiated carcinoma of the endometrium. Int J Gynecol Pathol 2009;28:142-147. 